\title{
The $\alpha$-HeliX AND The Organization AND Gating OF Channels
}

\author{
Robert H. Spencer ${ }^{1}$ and Douglas C. Rees ${ }^{2}$ \\ ${ }^{I}$ Department of Pharmacology, Merck Research Laboratories, West Point, \\ Pennsylvania 19486; e-mail: rob_spencer@merck.com \\ ${ }^{2}$ Division of Chemistry and Chemical Engineering 147-75CH, Howard Hughes Medical \\ Institute, California Institute of Technology, Pasadena, California 91125; \\ e-mail:dcrees@caltech.edu
}

Key Words ion channels, membrane proteins, transmembrane helices, conformational changes

- Abstract The structures of an increasing number of channels and other $\alpha$-helical membrane proteins have been determined recently, including the KcsA potassium channel, the MscL mechanosensitive channel, and the AQP1 and GlpF members of the aquaporin family. In this chapter, the orientation and packing characteristics of bilayerspanning helices are surveyed in integral membrane proteins. In the case of channels, $\alpha$-helices create the sealed barrier that separates the hydrocarbon region of the bilayer from the permeation pathway for solutes. The helices surrounding the permeation pathway tend to be rather steeply tilted relative to the membrane normal and are consistently arranged in a right-handed bundle. The helical framework further provides a supporting scaffold for nonmembrane-spanning structures associated with channel selectivity. Although structural details remain scarce, the conformational changes associated with gating transitions between closed and open states of channels are reviewed, emphasizing the potential roles of helix-helix interactions in this process.

\section{CONTENTS}

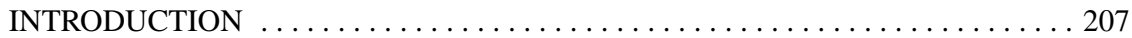

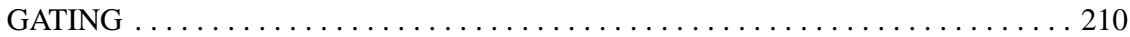

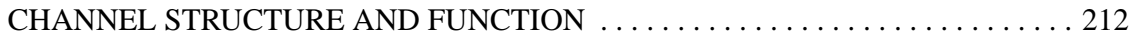

Characteristics of $\alpha$-Helices in Membrane Proteins $\ldots \ldots \ldots \ldots \ldots \ldots \ldots \ldots . \ldots \ldots 212$

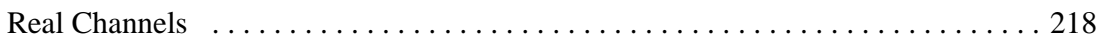

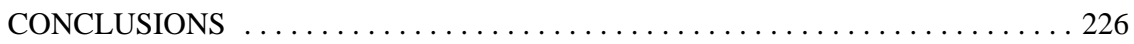

\section{INTRODUCTION}

Few discoveries have had a greater, or longer-lasting, impact on the field of protein structure and function than the $\alpha$-helix, first unveiled by Linus Pauling 50 years ago (74). This is particularly true in the study of membrane proteins, where the 
$\alpha$-helix provides an exquisite solution to the problem of satisfying the hydrogenbonding potential of the peptide bond in the apolar environment of the bilayer. (The $\beta$-sheet, Pauling's "other" secondary structure (73), also provides a solution to this problem adopted by outer-membrane proteins such as porins that are beyond the scope of this review.) Prior to any detailed structural understanding of membrane proteins or even of the membrane itself, it was anticipated that membrane-spanning proteins would be predominantly helical, based on observations that the $\alpha$-helix content of polypeptides increases significantly in nonaqueous solvents (87). These predictions were subsequently confirmed through Henderson \& Unwin's observation of seven rod-like features in the initial electron crystallographic studies of bacteriorhodopsin (37) and, ultimately, at high resolution in the structure of the photosynthetic reaction center by Deisenhofer et al. (18). Our present understanding of the primary and secondary structure organization of these integral membrane proteins can be summarized by the statements that the bilayer-spanning residues consist primarily of apolar amino acids and that these residues are predominantly found in the $\alpha$-helical conformation.

Channels constitute a functionally important class of membrane proteins that mediate the transmembrane passage of ions and other small molecules in their thermodynamically favorable direction [reviewed in $(1,38)$ ]. These macromolecular pores establish the basis for synaptic transmission, cell signaling, volume regulation, and many other critical physiological processes via their characteristic properties of ion selectivity, conductance, and gating. Channels may be highly selective for a particular molecular species (e.g., potassium, sodium, glycerol), or more permissive to a general class of molecules or ions such as anions, or they may even be completely nonselective. The structural basis for channel specificity has been established in greatest detail for the $\mathrm{K}^{+}$channel (22) and more recently for the aquaporin family $(30,66)$. The conductance of a channel specifies the number of molecules that can pass through the channel per unit time and depends on the geometry of the permeation pathway and the energetic profile for passage through the channel. In many cases, the conductance of a channel is regulated or gated by the conformational switching of the protein structure between "open" and "closed" states. The conformational sensitivity of channels to external influences is at the heart of the ability of these molecules to function as signal transducers. This behavior represents a fascinating biophysical challenge for understanding how the protein structure is coupled to these environmental cues.

Crystallographic analyses of the KcsA (22), MscL (11), AQP1 (66), and GlpF (30) channels have provided the first detailed views of their molecular organization (Figure 1). These channels represent a diverse cross section of channel activities: $\mathrm{KcsA}$ is a potassium ion-specific channel, MscL is a mechanosensitive channel that is most likely associated with protection against osmotic shock, and GlpF and AQP1 are members of the aquaporin family. Not coincidentally, the channels whose structures have been established by X-ray crystallography (KcsA, MscL, and GlpF) have all been isolated from prokaryotes, which reflects the great advantages (at present) of bacterial systems for the expression and purification of appropriate 

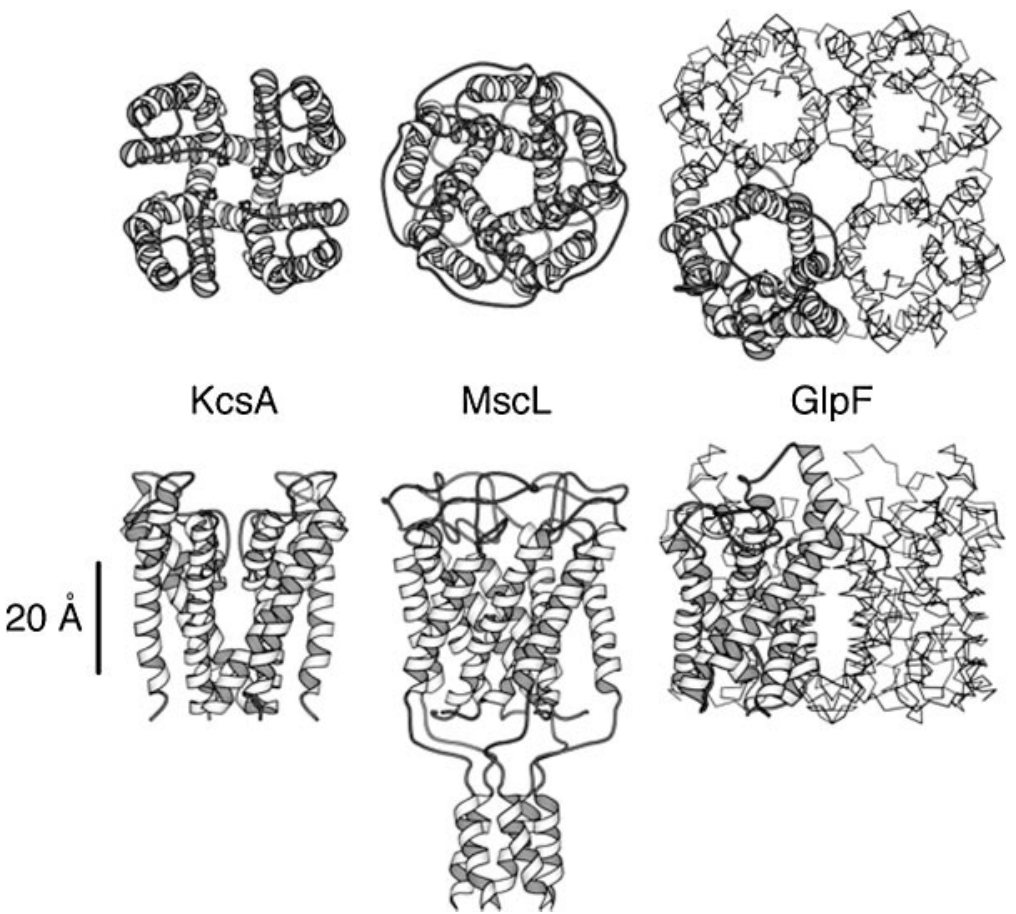

Figure 1 Structures of the KcsA (22), MscL (11), and GlpF (30) channels viewed parallel (top) and perpendicular (bottom) to the membrane normal. One of the four subunits in the GlpF tetramer is highlighted as a ribbons diagram in each view, with the $\mathrm{C} \alpha$ backbones of the remaining subunits traced in thinner lines. The vertical bar on the left indicates $20 \AA$. The molecular figures in this article were prepared with MOLSCRIPT (48).

quantities of naturally less-abundant membrane proteins for crystallization trials. As evidenced from the structures, the bilayer-spanning region of these channels is formed from a collection of $\alpha$-helices oriented such that the helix axes tend to be aligned along the membrane normal. The helices are packed together to form a sealed barrier that separates the hydrocarbon region of the bilayer from the permeation pathway for solutes. These structures provide a molecular framework for addressing functional properties of channels. For example, the structures of the KcsA and aquaporin family channels provide fundamental insights into the chemical basis for channel selectivity. In both systems, specificity is achieved through nonhelical structures in the center of the respective channels that form the "selectivity filter" responsible for selective binding of the appropriate molecular species. The structure of the KcsA channel has also permitted theoretical analyses of channel conductance that take into account the geometry of the permeation 
pathway, along with electrostatic and binding interactions that shape the energy landscape as the solute traverses the channel (81). Still to be established is the structural basis for the coupling of channel structure to environmental changes, in particular the issues of how the sensing occurs and how the protein conformation switchs between closed and open states. For channels such as MscL that have open states of large conductance, these conformational changes must be dramatic and clearly must involve rearrangements in the helix-helix packing interactions.

As these initial studies demonstrate, the $\alpha$-helix provides the central structural element for channel construction. This chapter explores implications of this $\alpha$-helical framework for the conformation and gating transitions of channels. After a brief introduction to gating, a more-detailed analysis of $\alpha$-helices in membranes will be presented, followed by a discussion of structurally characterized channels.

\section{GATING}

The simplest model for conformational gating treats the channel as exhibiting two conformational states, closed (C) and open (O)

$$
C \stackrel{K(V, c, P, e t c .)}{\leftrightarrow} O
$$

The equilibrium constant between these states is sensitive to the values of environmental parameters such as membrane potential (V), ligand concentrations (c), and tension applied to the bilayer $(\mathrm{P})$. By convention, this equilibrium is written such that the left-hand side is favored under resting conditions. For example, the closed state of $\mathrm{K}^{+}$channels and MscL are stabilized by $\sim 10 \mathrm{kcal} / \mathrm{mole}$ relative to the open state $(38,96)$. In response to appropriate changes in the environment, however, the equilibrium will shift toward the open state. Conformational changes between the closed and open states can formally be accomplished through many mechanisms. For voltage-gated channels, altered charge distributions between the open and closed states can couple protein conformation to changes in the membrane potential; for ligand-gated ion channels, changes in ligand affinity can shift the equilibrium between closed and open (or desensitized) states; for mechanosensitive channels, changes in membrane tension can differentially stabilize open and closed states that vary in cross-sectional areas. The steepness of the response provides a measure of the cooperative nature of the transition between states. Although this model clearly is an oversimplification of real systems, because detailed electrophysiological studies of ion channels have demonstrated the existence of multiple closed and open states, it does provide a useful basis for addressing the conformational basis of channel gating.

This description of channel gating may be treated within the framework of the Monod-Wyman-Changeux (MWC) model for allosteric systems $(12,65)$. In the MWC formalism, allosteric systems are considered to be composed of a symmetric arrangement of subunits that exist in two states, $\mathrm{R}$ and $\mathrm{T}$, with different properties; 
conventionally, the $\mathrm{R}$ and $\mathrm{T}$ states represent the active and inactive states. The equilibrium between these states may be shifted through the binding of ligands and other effectors that have different affinities for the two states:

$$
T \stackrel{1 / L}{\leftrightarrow} R .
$$

Typical values of the resting state ratio of $(\mathrm{R}) /(\mathrm{T})$ are in the range of $10^{-3}$ to $10^{-6}(33,76)$, so that the $\mathrm{T}$ state is favored in the absence of ligands by $\sim 5-10 \mathrm{kcal} / \mathrm{mole}$ - comparable to the energetic difference between open and closed states of channels. (Note, this equilibrium constant corresponds to the inverse of the allosteric constant L defined by MWC.) The binding of substrates and allosteric activators increases activity by shifting the equilibrium toward the more-active $\mathrm{R}$ state, whereas allosteric inhibitors stabilize the less-active $\mathrm{T}$ state. A critical insight from this model is that the cooperativity characteristic of allosteric systems reflects their oligomeric construction. A key assumption of the MWC treatment is that the oligomer remains symmetric, so that it behaves as a two-state system ( $\mathrm{R}$ or $\mathrm{T})$. This has the important consequence that the structure and ligand-binding properties of each subunit depend only on the quaternary state of the system and not on how many ligand molecules are bound. Again, although this model is overly simplistic, it does capture the essence of many real systems.

In view of the parallels between gated channels and allosteric enzymes, the general types of conformational changes associated with gating are anticipated to resemble those observed for allosteric systems $(33,61,76)$. Switching between the $\mathrm{R}$ and $\mathrm{T}$ states can involve changes in both quaternary and tertiary levels of structural organization. Quaternary structural changes result in alteration of subunit-subunit interactions, which can achieve global rearrangements that effectively propagate ligand-binding events over large distances from the ligand-binding sites. These changes typically involve switching between alternate close-packed and interdigitated configurations of the polypeptide chain at the subunit-subunit surfaces. Tertiary structural changes primarily involve rearrangements within subunits. Although these can be more localized than quaternary changes, alterations in domain-domain interfaces can also be comparable to rearrangements of subunitsubunit interfaces and consequently can involve large-scale rearrangements. Orderdisorder transitions that result in the folding or unfolding of part of the polypeptide chain have also been associated with allostery $(33,61)$. Many of the general types of gating models proposed for ion channels [see (38)] have counterparts in structurally characterized transitions in allosteric systems; for example, alterations in subunit-subunit interactions between closed and open states correspond to quaternary structure changes, whereas gating models descriptively designated "swinging door," "slider," "tethered-ball," and "ball-and-chain" involve tertiary structure changes.

Gerstein et al. (32) have identified and documented two major types of conformational changes that can occur within domains "hinge" and "shear." Hinge motions involve larger-scale movement in residues not constrained by packing interactions due to a few large changes in the main chain torsion angles of spatially 
adjacent residues, which represent the hinge. In view of the close-packed nature of membrane-spanning domains, hinge motions in channels would seem more relevant for conformational changes involving the extramembrane regions. In contrast, shear motions involve more-localized changes between close-packed segments that do not involve repacking of interfaces or deformation of the main chain. An important feature of proteins exhibiting shear motions is that they may be considered to have a "layered architecture," and conformational changes proceed through the sliding of adjacent layers. Of significance for our channel discussion, helices are an important component of these layers. The helices most commonly found in sliding interfaces are usually crossed, as opposed to more nearly (anti)parallel. Gerstein et al. (32) speculate that the smaller interface between crossed helices, relative to more parallel arrangements, can better accommodate the structural changes associated with shear motions.

\section{CHANNEL STRUCTURE AND FUNCTION}

\section{Characteristics of $\alpha$-Helices in Membrane Proteins}

As a reference point for our discussion of channel structure and function, some general characteristics of $\alpha$-helices in membrane proteins are briefly reviewed. This discussion builds extensively on Bowie's insightful analysis of helix-helix interactions in membrane proteins (8). Given the still small number of channel structures, the present analysis is based on a larger set of helical membrane protein structures, both channel and nonchannel, that contains 15 proteins with a total of 139 membrane-spanning helices (Table 1). Although the energetic principles driving the structural organization of membrane proteins are not explicitly discussed, engaging expositions of this relevant and fundamentally important topic may be found in recent reviews by White \& Wimley (105), Popot \& Engelman (77), and Fleming (27). A discussion of the consequences of deviations from noncanonical $\alpha$-helical geometries for membrane protein structure and function may be found in $(80 \mathrm{a}, 102)$.

To begin, we define a coordinate system for membrane proteins using the convention that the membrane normal is oriented along the $z$-axis, with the origin corresponding to the center of the membrane-spanning region and the positive $z$-axis pointing toward the outside of the cell (Figure 2). Because the membranespanning region cannot be experimentally defined, with the exception of a few proteins such as bacteriorhodopsin that have been studied by electron crystallography in their native membranes, it is necessary to identify this region through computational methods. For proteins that are oligomeric (including most of the proteins in Table 1), the direction of the rotation axis relating identical or homologous subunits is taken to correspond to the membrane normal. For the remaining structures, the direction of the membrane normal is defined such that the overall tilt of the helix axes from the normal is minimized. In this coordinate frame, the membrane-spanning region is assigned following the approach of Deisenhofer \& 
TABLE 1 Proteins used in the analyses of membrane-spanning helices that are described in the text

\begin{tabular}{|c|c|c|c|c|}
\hline PDB & $\mathbf{T M}$ & $\mathbf{N}$ & Protein & Ref. \\
\hline 1 occ & 28 & 2 & bovine cytochrome $c$ oxidase & $(101)$ \\
\hline 1qle & 22 & 1 & Paracoccus denitrificans cytochrome $c$ oxidase & (43) \\
\hline 1bgy & 13 & 2 & bovine cytochrome $b c_{1}$ & $(42)$ \\
\hline $2 \mathrm{prc}$ & 11 & 1 & Rhodopseudomonas viridis photosynthetic reaction center & $(18)$ \\
\hline 1aij & 11 & 1 & Rhodobacter sphaeroides photosynthetic reaction center & $(90)$ \\
\hline 1eul & 10 & 1 & rabbit calcium ATPase & $(99)$ \\
\hline $1 b r x$ & 7 & 3 & Halobacterium salinarium bacteriorhodopsin & $(59)$ \\
\hline $1 \mathrm{e} 12$ & 7 & 3 & halorhodopsin & $(47)$ \\
\hline $1 \mathrm{f} 88$ & 7 & 1 & bovine rhodopsin & $(69)$ \\
\hline $1 \mathrm{fx} 8$ & 6 & 4 & E. coli glycerol facilitator GlpF & $(30)$ \\
\hline 1 fum & 6 & 1 & E. coli fumarate reductase & $(41)$ \\
\hline 1qla & 5 & 2 & Wolinella succinogenes fumarate reductase & $(51)$ \\
\hline $1 \mathrm{kzu}$ & 2 & 9 & Rhodopseudomonas acidophila light harvesting complex & $(63)$ \\
\hline $1 \mathrm{~b} 18$ & 2 & 4 & Streptomyces lividans $\mathrm{K}^{+}$channel $\mathrm{Kcs} \mathrm{A}$ & $(22)$ \\
\hline $1 \mathrm{msl}$ & 2 & 5 & Mycobacterium tuberculosis mechanosensitive MscL & $(11)$ \\
\hline
\end{tabular}

PDB denotes identifier for the coordinates in the Protein Data Bank $(5,97)$; TM is the number of unique membrane-spanning helices in each structure; and $\mathrm{N}$ denotes the copy number of identical subunits in the likely functional unit.

Michel (19), who characterized the relative apolarity of the protein surface by the fraction of the accessible surface area contributed by carbon atoms. The origin is then assigned as the midpoint of the region with the greatest average surface apolarity.

As seen in Figure 3, helical membrane proteins exhibit a $20 \AA$-wide region with $>90 \%$ of the surface area contributed by carbon atoms. This same region is also characterized by the presence of few potentially charged side chains. Adjacent to this central region, the apolarity of the protein surface tapers down over a $10 \AA$-wide band to the $\sim 55 \%$ contribution typical of water-accessible surfaces, while the number of charged side chains increases sharply in this same region. Hence, based on surface apolarity, membrane proteins exhibit a $40 \AA$-wide band composed of a $20 \AA$-wide central region with flanking $10 \AA$-wide stripes that presumably correspond to the hydrocarbon and headgroup regions of the membrane bilayer. The $20 \AA$ width of the most apolar band of the membrane-spanning surface corresponds well with the region of minimum-charge density identified in experimentally determined electron-density distributions for membrane bilayers (105) and with the region of maximum probability for the distribution of carbon atoms in the hydrocarbon chains of phospholipids (67). These studies have also 


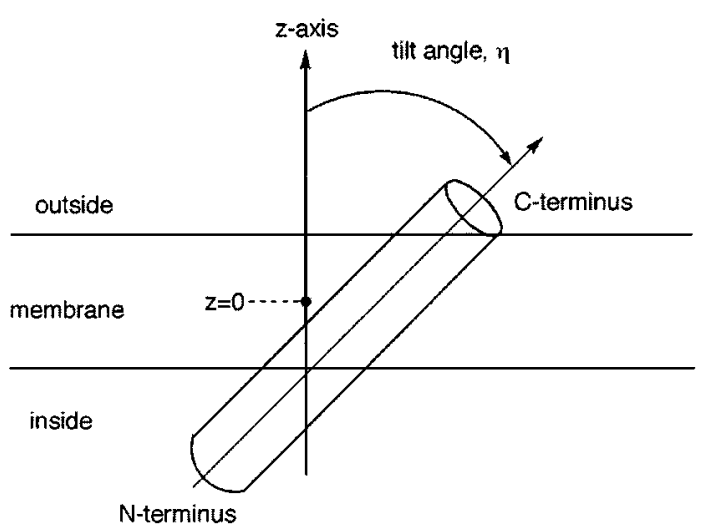

Figure 2 Definition of the coordinate system used in this article for the analysis of helix properties, including the orientation of the membrane normal along the $z$-axis, assignment of the membrane center to $z=0$, and the convention used to define the helix tilt angle, $\eta$.

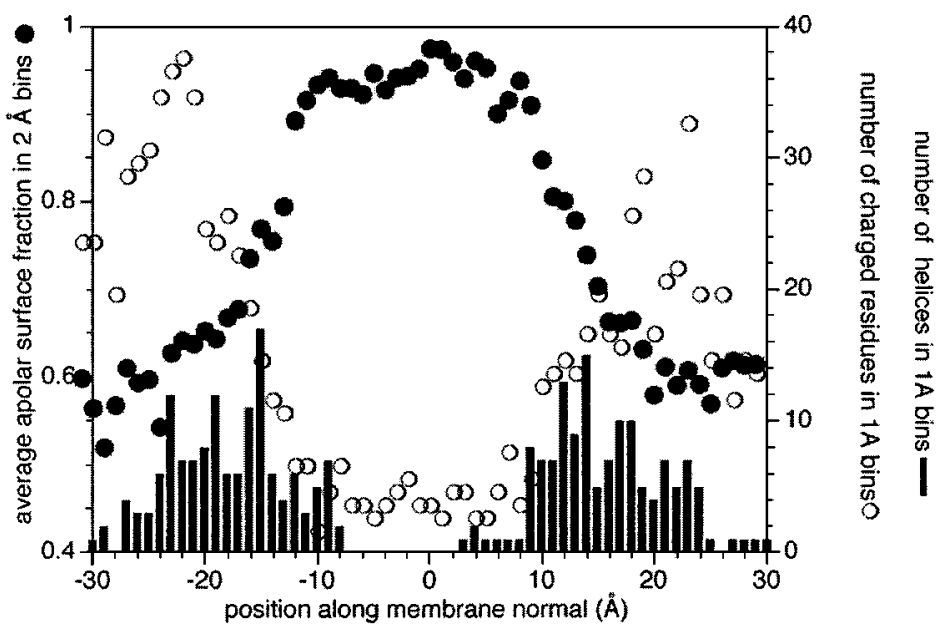

Figure 3 Variations with position in the apolarity of the protein surface and in the distribution of charged residues and helix termini, relative to the membrane, for the proteins listed in Table 1 . The fractions of the accessible surface area contributed by carbon atoms are indicated by filled circles $(\bullet)$, as calculated in $2 \AA$ slabs perpendicular to the membrane normal and averaged over all proteins. The numbers of side chains of potentially charged residues (Arg, Asp, Glu, His, and Lys) are represented by open circles (o), as calculated in $1 \AA$ intervals along $z$, while the histogram indicates the number of membrane-spanning helices that terminate in $1 \AA$ bins centered at the indicated $z$ value. The residues in each helix were identified with the program STRIDE (29). The positions of charged side chains were assigned from locations of the $\mathrm{C} \zeta, \mathrm{C} \gamma$, $\mathrm{C} \delta, \mathrm{C} \gamma$, and $\mathrm{N} \zeta$ atoms for Arg, Asp, Glu, His, and Lys, respectively. 
positioned the maximum of the probability distribution for the phosphate headgroups to be approximately $40 \AA$ apart, which coincides with the transition of the average polarity of the protein surface to that typical of water-soluble surfaces.

The high average hydrophobicity of the protein surface is consistent with the apolar nature of the lipid-exposed residues in integral membrane proteins. This hydrophobicity analysis may also be extended to include interior residues, by using surface area calculations to identify residues in the membrane-spanning helices that are either lipid-exposed (surface) or lipid-inaccessible (interior). For this calculation, residues were used that had $\mathrm{C} \alpha$ s positioned between $-10 \AA \leq z \leq 10 \AA$ in the membrane-spanning helices of the proteins in Table 1. Surface residues were identified as exposing at least $20 \%$ of the total surface area for a given type of amino acid. This classification resulted in 33\% and $67 \%$ of the residues assigned to the surface and interior classes. The average residue hydrophobicities of these two classes were evaluated with the hydrophobicity scale defined by Eisenberg et al. (24), where the extreme values are set by arginine and isoleucine at -1.76 and +0.73 . For the proteins in Table 1 , the average hydrophobicities of the interior and surface residues are calculated to be +0.23 and +0.41 . Hence, while the interior and surface residues of membrane proteins are, on average, both apolar, the lipid-exposed surface residues tend to be more apolar. Furthermore, the interior residues of membrane proteins have comparable hydrophobicities to the interior residues of water-soluble proteins. This analysis is completely consistent with observations reported on earlier membrane protein structures $(79,104)$. One implication of this behavior is that it should be possible to solubilize membrane proteins by replacement of lipid-exposed residues with polar amino acids (80); the design, preparation, and characterization of solubilized forms of phospholamban have recently been described $(28,57)$.

The positioning of bilayer-spanning helices within the membrane may be assessed from the locations of the helix termini with respect to the membrane coordinate system described in Figure 2. For the purposes of this analysis, the helix boundaries were assigned by the program STRIDE (29); occasional irregularities in helix geometries can lead to the assignment of two helices within one membrane-spanning stretch. The length of the 139 membrane-spanning helices in Table 1 averages $26 \pm 6$ residues. When the positions of the termini for the helices along the membrane normal are projected onto the apolarity distribution (Figure 3), the clear trend is that most helices span the $20 \AA$-wide apolar region and end in the headgroup regions (104). Few helices, however, span the complete $40 \AA$ distance across the membrane from one headgroup-aqueous interface to the other. Of significance for later discussion, the amino-terminal ends of 76 helices $\left(0.547=76 / 139 \equiv \mathrm{P}_{\mathrm{N}}\right)$ are positioned on the cytoplasmic side.

The axes of membrane-spanning helices are not randomly oriented within the membrane, but rather tend to be aligned perpendicular to the bilayer plane. Bowie (8) observed that, on average, the helix axes are tilted $\sim 21^{\circ}$ relative to the membrane normal. Similar trends are observed in the present survey, with the helix tilt averaging $23^{\circ} \pm 10^{\circ}$ when the direction of the polypeptide chain across the membrane is ignored. The tilt-angle distribution for the proteins in Table 1, 


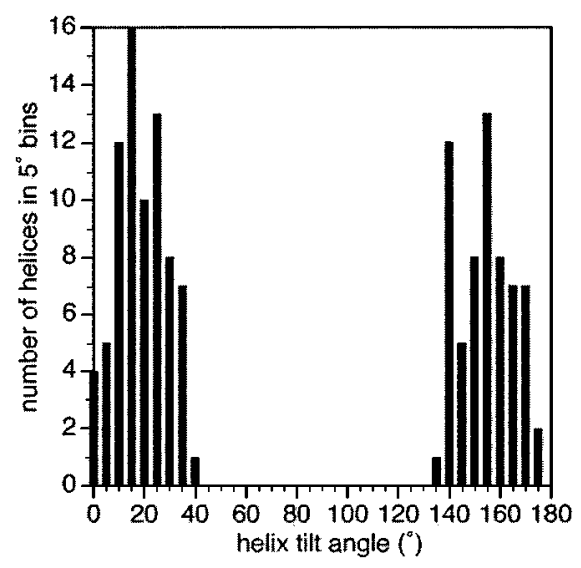

Figure 4 Histogram of the tilt angle of the helix axis with respect to the membrane normal for the proteins listed in Table 1 , tabulated in $10^{\circ}$ bins. Tilt angles between $0^{\circ}-90^{\circ}$ and $90^{\circ}-180^{\circ}$ correspond to helices with their $\mathrm{N}$-terminal ( 76 helices) or C-terminal (63 helices) ends closest to the cellular interior. For this calculation, the tilt angle was calculated from the transformation required to superimpose an ideal helix oriented along the $z$-axis onto those residues located within the $20 \AA$-wide apolar area (Figure 3) of membrane-spanning helices. Similar, but not identical, results (not shown) were obtained for the entire helical stretches owing to the presence of kinks and other irregular regions.

including the directionality of the polypeptide chain across the bilayer, is illustrated in Figure 4. Longer helices do not appear to be more (or less) tilted than their shorter counterparts (data not shown).

Both the interhelical-crossing angle and the distance of closest approach characterize the packing together of two helices (13). In this survey, these parameters were evaluated with the program PROMOTIF (40) for the $\alpha$-helical residues within the $20 \AA$-wide nonpolar surface region of the proteins listed in Table 1. As discussed by Bowie (8), helix-helix interactions with nearly parallel helix axes are much more favored in membrane proteins than in water-soluble proteins (Figure 5A). Parallel orientations result in more extensive helix-helix interfaces (7), which may contribute to the more efficient packing density that has been reported for membrane proteins $(23,31)$. A preference for small residues, particularly Gly, at helix interfaces $(23,44,54,82,83)$ should also contribute to the packing efficiency of membrane proteins.

Although the preference for helix axes to be parallel reflects the strong tendency of helices to be oriented along the membrane normal, it is not the entire story because left-handed packing arrangements are more abundant than right-handed ones $(8,52)$. Specifically, the observed distributions indicate an excess of left-handed interactions, with interhelical-crossing angles at $+20^{\circ}$ and $-160^{\circ}$ and a deficit of 
(A)

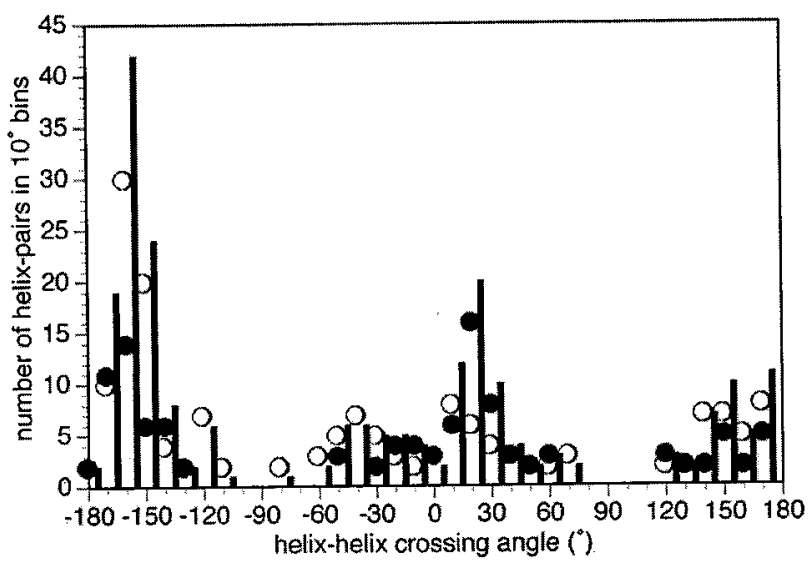

(B)

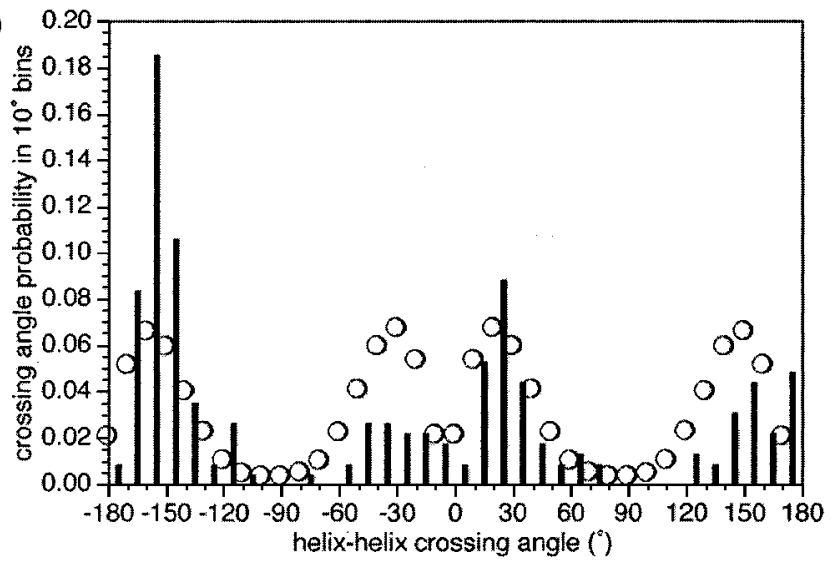

Figure 5 (A) Histogram of the 226 interhelical-crossing angles calculated with PROMOTIF (40) for the membrane-spanning helices in Table 1. The open (O) and closed $(\bullet)$ circles represent helical pairs separated by $<10 \AA$ (135 helices) and $>10 \AA$ (91 helices). The distributions were tabulated in $10^{\circ}$ bins, centered at the indicated values. Helical segments within the $20 \AA$-wide apolar region of the membrane (Figure 3) were used for this calculation; 21 of these helix contacts occurred between different protomers within an oligomer. $(B)$ Histogram of the observed interhelicalcrossing angle probability distribution with the expected (o) distribution superimposed, evaluated in $10^{\circ}$ bins. The observed distribution was obtained from the results of Figure $5 A$, divided by the total number of helical pairs in the data set (226 helices). The expected distribution was derived assuming the random pairing of helices obeying the tilt-angle distribution in Figure 4, allowing free rotation of the helices about the membrane normal. 
right-handed interactions at $-30^{\circ}$ and $+150^{\circ}$ relative to the frequencies expected (Figure $5 B$ ) for random pairings of helices with the observed tilt-angle distribution (Figure 4). In addition to the preference for left-handed packing arrangements, there is also a clear preference for antiparallel arrangements (8), which occur in $94 / 135=0.696$ of the helix pairs separated by less than $10 \AA$. This is significantly more frequent than expected from random considerations because, for the orientations of helices in this database, the expected fraction for antiparallel arrangements is calculated to be $0.496\left(=2 \times \mathrm{P}_{\mathrm{N}} \times\left(1-\mathrm{P}_{\mathrm{N}}\right)\right)$-close to that actually observed for helix pairs separated by more than $10 \AA(0.528=48 / 91)$.

In all channels characterized to date, the permeation pathway for solutes through the membrane is lined by helices. For symmetric, oligomeric proteins that have the permeation pathway generated by one helix per subunit, the tilt of the helix with respect to the membrane normal, $\eta$, the interhelical-crossing angle, $\alpha$, and the minimum pore radius, $\mathrm{R}_{0}$, are not all independent. With perfectly regular helices modeled as cylinders of diameter $d$ and exact $\mathrm{N}$-fold rotational symmetry, the variation in minimum pore radius with helix tilt may be shown to be

$$
R_{0}=\frac{d}{2}\left(\tan \eta \cot \left(\frac{\alpha}{2}\right)-1\right)
$$

where

$$
\cos \alpha=\cos ^{2} \eta+\sin ^{2} \eta \cos \theta
$$

and $\theta=2 \pi / \mathrm{N}$. The dependence of $\mathrm{R}_{0}$ on $\eta$ is illustrated in Figure 6 for different numbers of subunits in an oligomer. For perfectly regular helices and ideal oligomeric symmetry, an increase in pore radius requires an increase in helical tilt. At least for tetramers and pentamers, significant changes in pore radius only occur as the helix tilt exceeds $\sim 40^{\circ}$. Not surprisingly, for a given helix tilt, the pore radius increases with the number of helices lining the permeation pathway. It has been noted, for example, that pentameric channels tend to have larger pore diameters and poorer ion selectivity than channels formed from four subunits (38). This simple analysis further suggests that formation of a pore of any diameter is essentially impossible from three ideal helices.

\section{Real Channels}

LIGAND-GATED CHANNELS: THE NICOTINIC ACETYLCHOLINE RECEPTOR The nicotinic acetylcholine receptor (nAChR) isolated from the muscle-derived electric organ of the Torpedo ray provided the first visual evidence of the three-dimensional structure of an ion channel (100). Organized as a heteropentamer $\left(\alpha_{2} \beta \gamma \delta\right)$ of homologous subunits that are each predicted to contain four transmembrane segments (designated M1 to M4), this receptor is the classic representative of a superfamily of phylogenetically related proteins including the glycine, $\gamma$-aminobutyric acid, and serotonin receptors [reviewed in $(14,53)]$. Three-dimensional views of this receptor reconstructed from electron microscopic images, including the 


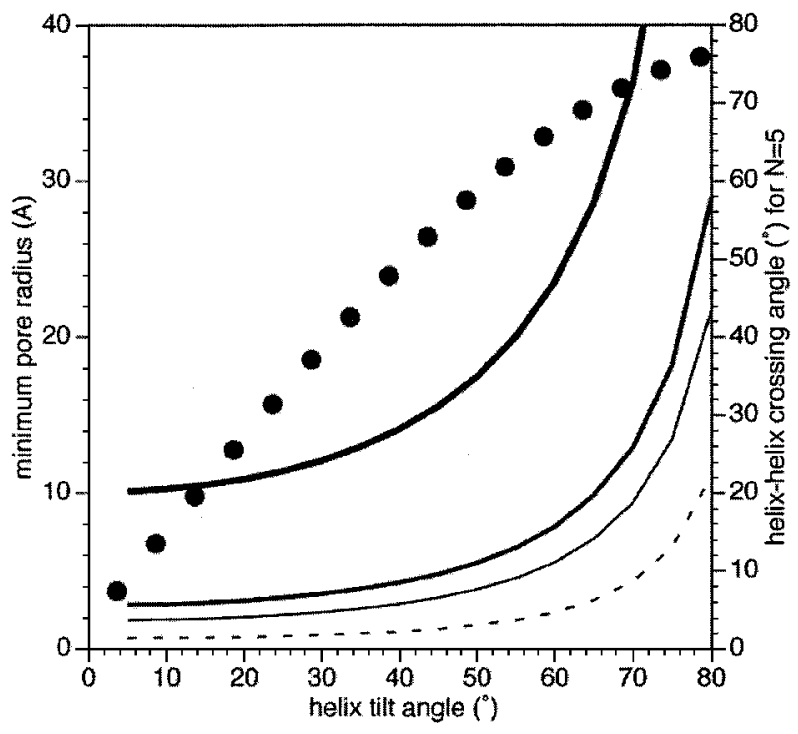

Figure 6 Dependence of the minimum pore radius $\mathrm{R}_{0}$ on the helix tilt angle for ideal, oligomeric channels with one helix per subunit lining the permeation pathway. $R_{0}$ values ( $\AA$, left-hand axis) are calculated as a function of tilt angle using Equations 1 and 2 for $\mathrm{N}=3$ (dotted line), 4 (thin line), 5 (medium line), and 10 (dark line). Circles (•, right-hand axis) indicate the interhelical-crossing angles calculated as a function of tilt angle for $\mathrm{N}=5$ from Equation 2. A helix diameter $d=9 \AA$ was used in these calculations.

recent analysis at $4.6 \AA$ resolution (64), provided the first structural evidence of an hourglass-shaped pore coursing through the central axis of the receptor complex, with the narrowest region localized approximately midway through the membrane. The permeation pathway is lined by five $\alpha$-helices that pack together in a right-handed bundle. Based upon a variety of data gathered from biochemical, electrophysiological, and mutagenesis experiments, the pore region is most probably lined by residues contributed by the M2 transmembrane segments from each subunit, with each M2 segment adopting an $\alpha$-helical structure. The constricted region of the channel has been proposed to function as the physical gate, perhaps formed by a thin ring of apolar side chains that regulate ionic flux through the transbilayer pore. Although the exact identities of the residues involved in forming the physical channel gate are uncertain, a series of conserved leucine residues have been implicated [(50); reviewed in (14)]. Consistent with the hypothesis that this constriction or kink in the M2 helices regulates the ion-conduction pathway, Unwin observed conformational changes within this region upon flash-freezing the receptor after brief exposure to acetylcholine (103). Evidence from cysteine mutagenesis studies differs from this interpretation 
(3), however, highlighting the need for further increases in the resolution of the current structural model.

The recent crystal structure determination of a soluble acetylcholine-binding protein (AChBP) from snails (9) represents an important advance in characterizing the binding of ligands in this system. AChBP forms a stable homopentamer of subunits homologous to the ligand-binding domain of nAChR; significantly, AChBP binds agonists and competitive antagonists of nAChR (36a). The AChBP subunit adopts an immunoglobin-like fold, with ligand-binding sites located at the subunit-subunit interfaces, sites that seemed to be blocked by the binding of a neurotoxin, $\alpha$-bungarotoxin (36a). From the location of a buffer molecule believed to serve as an acetylcholine mimic, the molecular details of the binding site have been defined. In particular, the binding site is rich in aromatic residues, as anticipated (14), that interact with quaternary amine-containing agonists through cation-pi interactions (21). The gating mechanism(s) by which ligand binding is coupled to changes in the transmembrane domain remain obscure, and future developments on this system are eagerly awaited.

SELECTIVITY AND VOLTAGE-GATING: $\mathrm{K}^{+}$CHANNELS The regulation and maintenance of an electrochemical potential across the plasma membrane are vital to all living cells and provide the energy required for driving many metabolic and cellular functions, most classically exemplified in the case of nerve and muscle firing. Voltage-sensitive $\mathrm{K}^{+}$channels $(\mathrm{Kv})$ not only play a critical role in controlling the resting membrane potential but also affect the duration and amplitude of signaling events (action potentials) based upon their gating and inactivation properties, as well as other factors (e.g., unitary conductance and level of expression). Due to the extensive study of $\mathrm{Kv}$ channels at the biophysical, biochemical, molecular, and atomic levels over the past decade and a half, we have gained a clearer understanding of structure-activity relationships within these proteins in regard to ion selectivity, subunit assembly, interactions with blockers, activation and gating, inactivation, and many other important questions.

Organized as tetramers of identical or related subunits $(60,86)$, the conduction pathway for $\mathrm{K}^{+}$ions runs parallel to the fourfold central axis of the channel, as clearly evidenced in the crystal structure of the prokaryotic $\mathrm{K}^{+}$channel, KcsA, by MacKinnon and coworkers (22). Each subunit within a Kv channel, as epitomized by the Shaker channel from Drosophila, is thought to have six membrane-spanning helices (designated S1 to S6), with the highly conserved interconnecting loop between S5 and S6 playing a predominant role in ion selectivity. The molecular organization of this region of the potassium channel was established by the structural analysis of KcsA (22). Each subunit of KcsA contains two membrane-spanning helices, corresponding to S5 and S6 in Kv channels, with the intervening segment containing the pore helix and a loop-forming structure, which functions as the selectivity filter. The permeation pathway through the membrane is formed by the second transmembrane helix (corresponding to S6). These helices are tilted $\sim 35^{\circ}$ from the membrane normal, and pack against their counterparts from 


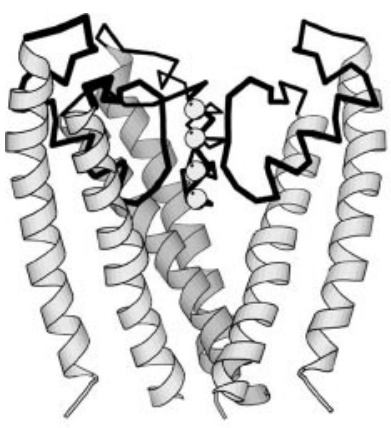

KcsA

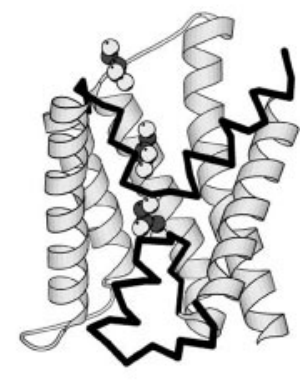

GIpF

Figure 7 Structures of KcsA (left) (22) and GlpF (right) (30) emphasizing the location of nonmembrane-spanning elements responsible for channel selectivity. The transmembrane helices are represented as ribbons, whereas the nonmembrane-spanning regions are depicted as solid traces. The positions of three potassium and one water in the selectivity filter of KcsA (left) and three glycerol molecules in the permeation pathway of GlpF (right) are indicated by CPK models. For clarity, one subunit of the KcsA tetramer has been removed, while only one subunit of GlpF is displayed, with the first and last transmembrane helices omitted.

adjacent subunits to form a right-handed bundle with interhelical-crossing angles of $-51^{\circ}$. The S5 and S6 helices provide the framework that positions the residues in the interconnecting loops responsible for ion selectivity. Significantly, the KcsA structure has provided physical evidence for the mechanism of ion selectivity, illustrating the coordination of dehydrated $\mathrm{K}^{+}$ions by backbone carbonyl atoms within the selectivity filter (22) (Figure 7). Based on the sequence similarity with the Kv channels, the structure of the KcsA channel most probably serves as a good model for the structure of the pore region in $\mathrm{Kv}$ channels.

Although we do not yet have a complete crystallographic model of a Kv channel, several pieces of structural information provide other clues as to some of the pertinent functional elements of the extramembrane components. A region $\mathrm{N}$-terminal to the first transmembrane helix of the $\mathrm{Kv}$ channels, known as $\mathrm{T} 1$, although not absolutely required for channel function (45), appears to participate in the specificity of subunit assembly $(58,85)$ and is also involved in the docking with modulatory $\mathrm{Kv} \beta$-subunits $(35,84)$. The tetrameric structure of the isolated T1 domain suggests that the respective T1 regions from each subunit might coassemble together below the cytoplasmic face of the channel pore (49). However, it was initially unclear how this structure might be compatible with biophysical data on the accessibility of the N-terminal "inactivation ball" to the cytoplasmic side of the pore. In support of this model, the structure of the T1 domain together with a modulatory $\beta$-subunit, which is known to contain an inactivation ball, has recently been solved (35). By combining this and other structural information 
with biochemical and biophysical evidence, it is suggested that the inactivation particle may gain access to the pore through a gap or window between the T1 and transmembrane domains $(35,46,88,106)$. Following initial interaction with the cytoplasmic channel surface, it is proposed that the inactivation particle subsequently enters the pore as an extended peptide and binds in a cavity adjacent to the selectivity filter, thereby blocking the channel (108). Because the overall quaternary structure appears to remain intact, these gating transitions are based on changes in the tertiary structure of the channel.

Although it is uncertain as to whether the subunit associations in the T1 domain rearrange or dissociate during channel gating in vivo, locking this domain together does not greatly affect channel gating (46). Additionally, replacing acidic residues within the linker region between the transmembrane and T1 domains (S1-T1) with neutral or basic residues decreases the rate of inactivation apparently by altering electrostatic interactions with the inactivation gate (35). The inactivation gate and several small-molecule inhibitors physically interact with the cytoplasmic side of the transmembrane pore $(20,108)$; because the opening through the center of the assembled T1 domain is too small and too basic to accommodate these molecules, the experimental evidence seems to support the model of lateral openings leading from the cytoplasm to the channel pore. Intriguingly, structural information for the ligand-gated and mechanosensitive channels also appear to exhibit similar openings beneath their respective transmembrane pores $(11,64)$, suggesting that this structural feature has been conserved throughout ion channel evolution and may play a valuable functional role.

Based on abundant electrophysiological and spectroscopic data, the transduction of membrane potential changes to conformational movements in Kv channels is likely mediated by the fourth transmembrane helix (S4), the "voltage-sensor," which contains a regular series of basic residues repeated at every fourth position. Additionally, negatively charged residues within the second and third transmembrane helices (S2 and S3) have been demonstrated to form salt bridges with the basic amino acids in the S4 helix, suggesting that these interactions stabilize and coordinate the structural rearrangements between the closed and open states $(26,70,98)$. In order to better understand the dynamics involved in the movement of the S4 helix during activation gating, a multitude of techniques have been employed [reviewed in $(6,39)$ ] and together suggest that during activation there is a rotation of the $\mathrm{S} 4$ helix and also a change in its helical tilt angle relative to the membrane. However, the mechanics involved in transducing movements in the voltage sensor into physical removal of a barrier within the ion permeation pathway is still unknown. This process could involve separation of the S5 and S6 helices at the base of the pore, as suggested in the pH-dependent gating of KcsA (16). At the current level of understanding, these alterations may be the consequence of shear-type motions (32) that result in the repositioning of helices around the pore.

SELECTIVITY AND FACILITATED DIFFUSION: AQUAPORINS The flux of water and other small solutes across the membrane must be carefully controlled to maintain the appropriate osmotic balance within the cell. Members of the aquaporin 
(AQP) family are widely distributed through a diverse set of organisms and include highly specific water-conducting channels [reviewed in (25)], as well as glycerolconducting channels such as the Escherichia coli glycerol facilitator, GlpF. Organized as tetramers of identical subunits, the quaternary structure of the AQP proteins seems reminiscent to that of the $\mathrm{K}^{+}$channel proteins. However, unlike the $\mathrm{K}^{+}$channels, each AQP subunit within the tetramer is capable of functioning as an independent channel pore. The conductance of some members of the AQP family are regulated by $\mathrm{pH}$ or phosphorylation.

The amino acid sequences of AQP family members show clear evidence for sequence duplication between the amino- and carboxyl-terminal halves of the channel, including a characteristic Asn-Pro-Ala (NPA) signature sequence found in each segment. Structures of the AQP family have recently been determined for the E. coli GlpF channel by X-ray crystallography at $2.2 \AA$ resolution (30) and for the red blood cell AQP1 by electron microscopy at $3.8 \AA$ resolution (66). These studies reveal that each subunit has six transmembrane helices threaded across the membrane, along with two half-helices (Figure 1). The membrane-spanning helices are tilted by an average of $\sim 33^{\circ}$ and form a right-handed helical bundle that provides the supporting scaffold for the two half-helices that enter from each side of the bilayer. The sequence duplication is reflected in the presence of an internal quasi-twofold axis in the plane of the membrane that relates the two structurally equivalent parts of the channel. In particular, the two conserved NPA motifs interact through the prolines across the interface surrounding this axis.

An exciting aspect of the GlpF structure is the presence of three glycerol molecules that identify the permeation pathway and establish the selectivity mechanism of this channel for glycerol (30). The challenge faced by the GlpF channel is to allow the passage of glycerol while absolutely discriminating against all ions including $\mathrm{OH}^{-}$and $\mathrm{H}^{+}$(67a). The channel conducts both larger and smaller solutes that are chemically similar to glycerol but with different conductances that reflect stereo- and enantioselectivity (30). As noted by the authors, this is not unlike the challenge faced by potassium channels that must selectively allow the passage of dehydrated potassium ions through its pore. The half-membrane-spanning helices in GlpF play a key role in its selectivity, particularly the Asn residues in the NPA motifs at the amino-terminal end of each helix, as well as the residues in the loop leading into these helices. Analogous to the KcsA channel, these residues can be considered to form the selectivity filter of the GlpF channel (Figure 7). Carbonyl groups from residues in this region form a hydrogen-bonding network on one side of the channel that can interact with the $\mathrm{OH}$ groups of glycerol and associated water molecules. The opposing side of the permeation pathway is lined with apolar groups that can participate in van der Waals contacts with the carbon atoms of glycerol. The amphipathic nature of the surface of the permeation pathway helps to select molecules, including the linear poly-alcohols termed alditols, that are similar to glycerol and share the characteristic of having a polar side and an apolar side. Furthermore, the apolar component of the permeation pathway likely serves to discriminate against ions and other charged molecules. Finally, the tight packing of this region prevents expansion of the pore necessary to permit the passage 
of larger molecules. As with the $\mathrm{K}^{+}$channel, the overall selectivity of aquaporin channels appears to reflect an energetic balance between competing repulsive and attractive forces.

MECHANOSENSITIVE CHANNELS: MscL The typically higher concentration of osmolytes within a bacterial cell relative to the surrounding environment provides a driving force for water flux into the cytoplasm. The resultant increase in volume of the cytoplasm creates a turgor pressure that counteracts this flux due to the expansion of the cytoplasmic membrane against the cell wall. Moderate turgor pressure provides the mechanical force for the expansion of the cell wall and is critical for bacterial growth. However, upon a sudden decrease in the osmolarity of the surrounding medium, water rapidly moves into the cells, increasing the turgor pressure to the point of cell rupture. Based upon the work of Kung et al. and others, it appears that nearly all eubacteria express mechanosensitive channels within the cytoplasmic membrane $(4,55,94)$ that are sensitive to membrane tension, and more recent work extends these observations to archaea (36).

The prokaryotic MscL, or mechanosensitive channel of large-conductance, provides a simplified paradigm for ion channels specialized in the transduction of mechanical stimuli (e.g., sound, touch, gravity, pressure) into an electrochemical response. First characterized by Kung et al. in E. coli following isolation of a stretch-activated channel, MscL exhibits a large conductance of $\sim 2.5 \mathrm{nS}$ with no ionic selectivity and was subsequently cloned and shown to function as a homomultimer (93). The crystal structure of MscL from Mycobacterium tuberculosis (11) provided clear evidence of the pentameric structure of this channel and demonstrated that the protein is organized as two helical domains, one intramembrane and one cytoplasmic (Figure 1). The membrane domain is composed of ten transmembrane helices, two from each subunit, whereas the cytoplasmic domain is organized as a left-handed bundle containing one helix from each subunit. The transmembrane helices are tilted by $\sim 35^{\circ}$, with respect to the membrane normal with the amino-terminal-most helix of each subunit (the inner helix) lining the permeation pathway. These inner helices pack together in a right-handed fashion with a crossing angle of $\sim-43^{\circ}$ to form the pore. This arrangement of helices around the permeation pathway is similar to that observed for KcsA, despite the opposite threadings of the polypeptide chains through the membranes, and the differences in oligomeric states (78). A generally consistent view of the MscL channel organization has been provided by recent site-directed spin label studies (75). The narrowest region of the pore occurs near the cytoplasmic side of the membrane domain, where the side chain of $\mathrm{Val}^{21}$ is positioned directly into the ionconduction pathway, in effect, occluding permeation of solutes through the pore. A critical role for $\mathrm{Val}^{21}$ in the gating of $\mathrm{MscL}$ has been demonstrated through mutagenesis studies (68). Other residues flanking this region, as well as more distantly located positions such as in the extracellular loop, have also been shown to have modulatory effects on the gating mechanism $(2,34,62,94,107)$, which may reflect their roles in the transition to the open state (91). 
Although significant structural rearrangements are necessary to account for the conductance properties of MscL in the open state, it is not obvious how the transmembrane and cytoplasmic domains structurally rearrange upon sensing membrane tension. With an estimated pore diameter of $\sim 30-40 \AA$ (15), the conformational changes associated with the closed-to-open transition of MscL must be substantial. Assuming that all the subunits in the channel are equivalent in the open state, an increase in pore radius necessarily requires some combination of an increased number of helices lining the pore and/or an increased tilt of helices lining the pore (Figure 6). An early model for the open state of MscL is based on the former possibility and invokes an alteration of inner and outer helices to create a 10-helix barrel-stave-type channel with the $\alpha$-helices nearly parallel to the membrane normal $(15,89)$. The conformational rearrangement associated with this transition could plausibly involve the coordinated movement of adjacent pairs of inner and outer helices that are nearly antiparallel because these types of helixhelix interfaces tend to be more stable (32). Attempts to generate explicit models of this type, however, have suggested that a substantial number of hydrophobic residues may be exposed to water in the open state, which would be energetically unfavorable (92). An alternative mechanism for increasing the number of helices, by increasing the number of subunits in the oligomer, appears unlikely in view of the observations of Sukharev et al. that the MscL oligomer is not in dynamic equilibrium with monomers (95).

Recently, a detailed proposal for the open state of MscL has been developed by Sukharev \& Guy, based on modeling studies combined with disulfide trapping and electrophysiological characterizations $(91,92)$. In this model (Figure 8), the symmetry of MscL is assumed to follow MWC-type postulates and is conserved in both closed and open states. The structural rearrangements for forming a large $\sim 30 \AA$ pore in the open state involve increasing the helical tilt from $35^{\circ}$ (closed) to $70^{\circ}$ (open); the inner helix lines the permeation pathway in both states. In the open-state model, the interhelical-crossing angle between adjacent inner helices increases from $-43^{\circ}$ to $-68^{\circ}$. Both the tilt and the helix crossing angle are essentially unprecedented in structurally characterized membrane proteins (Figures 4 and $5 A$ ), although of course, they are proposed to exist in a form that is significantly less stable than the closed state under resting conditions. Rather than the side chains of $\mathrm{Val}^{21}$ in the inner helices serving as the gate, Sukharev \& Guy propose that the real gate is a five-helix bundle containing the highly conserved aminoterminal residues of each subunit. These residues are not observed in the crystal structure, however, presumably due to disorder or other types of conformational heterogeneity. Experimental support for the Sukharev \& Guy model is provided by the ability to stabilize an open state of MscL through disulfide-bond formation between specifically incorporated cysteines that should be spatially adjacent only in the open state. Due to the role of protein dynamic effects (10) and the influence of the membrane location of the cysteines on the rate of disulfide formation (17), however, it would appear difficult to derive quantitative distance constraints from these experiments. Nevertheless, this general approach has many attractive 

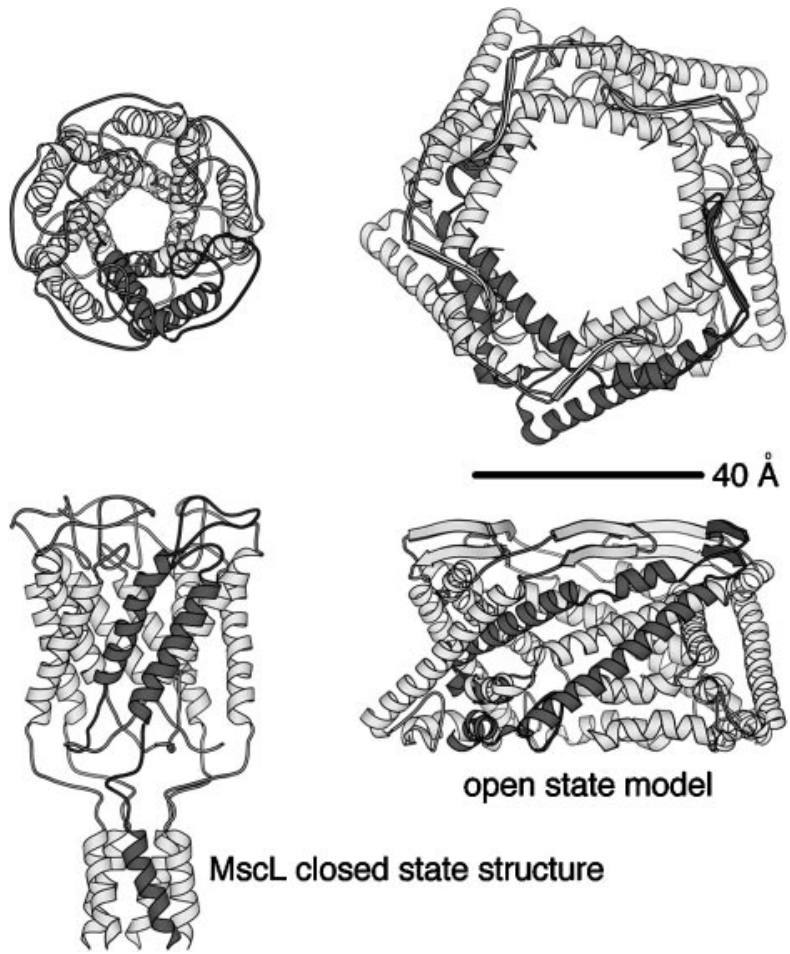

open state model

MscL closed state structure

Figure 8 Comparisons of the structures of MscL as observed crystallographically in the closed state (left) (11) and as modeled in the open state by Sukharev \& Guy (right) $(91,92)$. Views down the membrane normal (top) and in the plane of the membrane (bottom) are provided. One subunit is highlighted in each structure for clarity. The increased tilt of the membrane-spanning helices in the open-state model, along with the increased helical length necessary to span the bilayer in the open-state model, are evident. The dark horizontal line (right) indicates $40 \AA$.

features that could ultimately help lead to preparation and characterization of a stable open state for structural studies.

\section{CONCLUSIONS}

The $\alpha$-helix unquestionably serves as the dominant element in the structural organization of the membrane-spanning region of channels and many other proteins. Generalizing from the presently available structures of the KcsA, MscL, AQP1, and GlpF channels, as well as the overall properties of helices in membrane proteins, 
it appears that

1. The most-nonpolar region of the membrane-exposed surface is $20 \AA$ wide (Figure 3), compared to the overall thickness of the bilayer $(\sim 40 \AA)$, which should have implications for the electrostatic barrier to movement of ions across the membrane [for examples, see $(56,71,72)$ ].

2. The permeation pathways of channels are formed from helices that are tilted $\sim 30^{\circ}-40^{\circ}$ with respect to the membrane normal, which is relatively steep for membrane-spanning helices (Figure 4). The pore-forming helices are packed in right-handed bundles with a relatively steep interhelical-crossing angle $\left(\sim-40^{\circ}\right)$ (Figure $5 A$ ) that creates a funnel-like architecture with a defined point of constriction.

3. The determinants of channel selectivity are provided by nonmembranespanning elements supported within the helical bundle (Figure 7).

4. The conformational changes associated with channel gating can involve pronounced quaternary structure rearrangements of the pore-forming helices, as well as changes in tertiary structure involving these helices and extramembrane components of channels. At the high tilt angles observed for the pore-forming helices, the minimum pore diameter is particularly sensitive to changes in helix tilt (Figure 6).

The role of the $\alpha$-helix as the workhorse for ion channels reflects both the common elements of this motif, which are determined by the properties of the polypeptide backbone, as well as the infinite variations that can be incorporated through the particular choice of side chains dictated by the amino acid sequence. Hence, the same basic structural framework can be tailored for a myriad of specific functional purposes. From the available structures, common themes are emerging concerning the role of $\alpha$-helices in the organization of the resting states of various channels. The greatest outstanding challenges in our structural understanding of channels are to realize how rearrangements of this basic framework can be triggered and the mechanisms by which these changes occur.

\section{ACKNOWLEDGMENTS}

Discussions with Randal Bass, Kaspar Locher, Pavel Strop, Allen Lee, Margaret Barclay, Dennis Dougherty, Henry Lester, and Chris Miller are greatly appreciated. This work was supported in part by NIH grant GM62532.

\section{NOTE ADDED IN PROOF}

Recent advances in the structural characterization of channels and transporters have been provided by the crystal structure determinations of a KcsA potassium channel-Fab complex [(108a); with an accompanying analysis of the energetics of ion conductance $(5 \mathrm{a}, 65 \mathrm{a})]$ and of the E. coli MsbA transporter, a member of 
the ATP-binding cassette family (10a). Movements of the inner helices between the closed and open states of KcsA have been characterized by site-directed spin labeling studies (58a). The X-ray crystal structure of AQP1 has been reported at $2.2 \AA$ resolution $(90 \mathrm{a})$.

\section{Visit the Annual Reviews home page at www.annualreviews.org}

\section{LITERATURE CITED}

1. Aidley DJ, Stanfield PR. 1996. Ion Channels. Cambridge: Cambridge Univ. Press. 307 pp.

2. Ajouz B, Berrier C, Besnard M, Martinac B, Ghazi A. 2000. Contributions of different extramembranous domains of the mechanosensitive ion channel MscL to its response to membrane tension. $J$. Biol. Chem. 275:1015-22

3. Akabas MH, Kaufmann C, Archdeacon P, Karlin A. 1995. Identification of acetylcholine receptor channel-lining residues in the entire $\mathrm{M} 2$ segment of the alpha subunit. Neuron 13:919-27

4. Batiza AF, Rayment I, Kung C. 1999. Channel gate! Tension, leak and disclosure. Structure 7:R99-103

5. Berman HM, Westbrook J, Feng Z, Gilliland G, Bhat TN, et al. 2000. The Protein Data Bank. Nucleic Acids Res. 28:235-42

5a. Bernèce S, Roux B. 2001. Energetics of ion conduction through the $\mathrm{K}^{+}$channel. Nature 414:73-77

6. Bezanilla F. 2000. The voltage sensor in voltage-dependent ion channels. Physiol. Rev. 80:555-92

7. Bowie JU. 1997. Helix angle packing preferences. Nat. Struct. Biol. 4:915-17

8. Bowie JU. 1997. Helix packing in membrane proteins. J. Mol. Biol. 272:780-89

9. Brejc K, van Dijk WJ, Klaassen RV, Schuurmans M, van der Oost J, et al. 2001. Crystal structure of an ACh-binding protein reveals the ligand-binding domain of nicotinic receptors. Nature 411:269-76

10. Butler SL, Falke JJ. 1996. Effects of pro- tein stabilizing agents on thermal backbone motions: a disulfide trapping study. Biochemistry 35:10595-600

10a. Chang G, Roth CB. 2001. Structure of MsbA from E. coli: a homolog of the multidrug resistance ATP binding cassette (ABC) transporters. Science 293: 1793-800

11. Chang G, Spencer RH, Lee AT, Barclay MT, Rees DC. 1998. Structure of the MscL homolog from Mycobacterium tuberculosis: a gated mechanosensitive ion channel. Science 282:2220-26

12. Changeux JP, Edelstein SJ. 1998. Allosteric receptors after 30 years. Neuron 21:959-80

13. Chothia C, Levitt M, Richardson D. 1981. Helix to helix packing in proteins. J. Mol. Biol. 145:215-50

14. Corringer P-J, Le Novere N, Changeux J-P. 2000. Nicotinic receptors at the amino acid level. Annu. Rev. Pharmacol. Toxicol. 40:431-58

15. Cruickshank CC, Minchin RF, LeDain AC, Martinac B. 1997. Estimation of the pore size of the large-conductance mechanosensitive ion channel of Escherichia coli. Biophys. J. 73:1925-31

16. Cuello LG, Romero RG, Cortes DM, Perozo E. 1998. pH-dependent gating in the Streptomyces lividans $\mathrm{K}^{+}$channel. Biochemistry 37:3229-36

17. Czerski L, Sanders CR. 2000. Thiol modification of diacylglycerol kinase: dependence upon site membrane disposition and reagent hydrophobicity. FEBS Lett. 472:225-29

18. Deisenhofer J, Epp O, Miki K, Huber R, 
Michel H. 1985. Structure of the protein subunits in the photosynthetic reaction center of Rhodopseudomonas viridis at $3 \AA$ resolution. Nature 318:618-24

19. Deisenhofer J, Michel H. 1989. The photosynthetic reaction centre from the purple bacterium Rhodopseudomonas viridis. EMBO J. 8:2149-70

20. del Camino D, Holmgren M, Liu Y, Yellen G. 2000. Blocker protection in the pore of a voltage-gated $\mathrm{K}^{+}$channel and its structural implications. $\mathrm{Na}$ ture 403:321-25

21. Dougherty DA. 1996. Cation-pi interactions in chemistry and biology: a new view of benzene, phe, tyr and trp. Science 271:163-68

22. Doyle DA, Cabral JM, Pfuetzner RA, Kuo A, Gulbis JM, et al. 1998. The structure of the potassium channel: molecular basis of $\mathrm{K}^{+}$conduction and selectivity. Science 280:69-77

23. Eilers M, Shekar SC, Shieh T, Smith SO, Fleming PJ. 2000. Internal packing of helical membrane proteins. Proc. Natl. Acad. Sci. USA 97:5796-801

24. Eisenberg D, Weiss RM, Terwilliger TC, Wilcox W. 1982. Hydrophobic moments and protein structure. Faraday Symp. Chem. Soc. 17:109-20

25. Engel A, Fujiyoshi Y, Agre P. 2000. The importance of aquaporin water channel protein structures. EMBO J. 19:800-6

26. Fedida D, Hesketh J. 2001. Gating of voltage-dependent potassium channels. Prog. Biophys. Mol. Biol. 75:165-99

27. Fleming KG. 2000. Riding the wave: structural and energetic principles of helical membrane proteins. Curr. Opin. Biotechnol. 11:67-71

28. Frank S, Kammerer RA, Hellstern S, Pegoraro S, Stetefeld J, et al. 2000. Toward a high-resolution structure of phospholamban: design of soluble transmembrane domain mutants. Biochemistry 39:6825-31

29. Frishman D, Argos P. 1995. Knowledgebased protein secondary structure as- signment. Proteins Struct. Funct. Genet. 23:566-79

30. Fu D, Libson A, Miercke LJW, Weitzman C, Nollert P, et al. 2000. Structure of a glycerol-conducting channel and the basis for its selectivity. Science 290:48186

31. Gerstein M, Chothia C. 1999. Proteins in motion. Science 285:1682-83

32. Gerstein M, Lesk AM, Chothia C. 1994. Structural mechanisms for domain movements in proteins. Biochemistry 33:6739-49

33. Goldsmith EJ. 1996. Allosteric enzymes as models for chemomechanical energy transducing assemblies. FASEB $J$. 10:702-8

34. Gu LQ, Liu WH, Martinac B. 1998. Gating pattern for the G14E site-directed mutant of the large mechanosensitive ion channel (MscL) of Escherichia coli characterized by a continuous spectrum of subconductance levels. Biophys. J. 74:A324

35. Gulbis JM, Zhou M, Mann S, MacKinnon R. 2000. Structure of the cytoplasmic $\beta$ subunit- $\mathrm{T} 1$ assembly of voltage-dependent $\mathrm{K}^{+}$channels. Science 289:123-27

36. Hamill OP, Martinac B. 2001. Molecular basis of mechanotransduction in living cells. Physiol. Rev. 81:685-740

36a. Harel M, Kasher R, Nicolas A, Guss JM, Balass M, et al. 2001. The binding site of acetylcholine receptor as visualized in the X-ray structure of a complex between $\alpha$-bungarotoxin and a mimotope peptide. Neuron 32:265-75

37. Henderson R, Unwin PNT. 1975. Threedimensional model of purple membrane obtained by electron microscopy. Nature 257:28-32

38. Hille B. 2001. Ionic Channels of Excitable Membranes. Sunderland, MA: Sinauer. 853 pp.

39. Horn R. 2000. A new twist in the saga of charge movement in voltage-dependent ion channels. Neuron 25:511-14 
40. Hutchinson EG, Thornton JM. 1996. PROMOTIF - a program to identify and analyze structural motifs in proteins. Protein Sci. 5:212-20

41. Iverson TM, Luna-Chavez C, Cecchini G, Rees DC. 1999. Structure of the Escherichia coli fumarate reductase respiratory complex. Science 284:1961-66

42. Iwata S, Lee JW, Okada K, Lee JK, Iwata M, et al. 1998. Complete structure of the 11-subunit bovine mitochrondrial cytochrome $b c_{1}$ complex. Science 281: 64-71

43. Iwata S, Ostermeier C, Ludwig B, Michel H. 1995. Structure at $2.8 \AA$ resolution of cytochrome $c$ oxidase from Paracoccus denitrificans. Nature 376: 660-43

44. Javadpour MM, Eilers M, Groesbeek M, Smith SO. 1999. Helix packing in polytopic membrane proteins: role of glycine in transmembrane helix association. Biophys. J. 77:1609-18

45. Kobertz WR, Miller C. $1999 . \mathrm{K}^{+}$channels lacking the 'tetramerization' domain: implications for pore structure. Nat. Struct. Biol. 6:1122-25

46. Kobertz WR, Williams C, Miller C. 2000. Hanging gondola structure of the $\mathrm{T} 1$ domain in a voltage-gated $\mathrm{K}^{+}$channel. Biochemistry 39:10347-52

47. Kolbe M, Besir H, Essen L-O, Oesterhelt D. 2000. Structure of the light-driven chloride pump halorhodopsin at $1.8 \AA$ resolution. Science 288:1390-96

48. Kraulis PJ. 1991. Molscript-a program to produce both detailed and schematic plots of protein structures. J. Appl. Crystallogr. 24:946-50

49. Kreusch A, Pfaffinger PJ, Stevens CF, Choe S. 1998. Crystal structure of the tetramerization domain of the Shaker potassium channel. Nature 392:94548

50. Labarca C, Nowak M, Zhang H, Tang L, Deshpande P, Lester H. 1995. Channel gating governed symmetrically by conserved leucine residues in the M2 domain of nicotinic receptors. Nature 376:514-16

51. Lancaster CRD, Kröger A, Auer M, Michel H. 1999. Structure of fumarate reductase from Wolinella succinogenes at $2.2 \AA$ resolution. Nature 402:377-85

52. Langosch D, Heringa J. 1998. Interaction of transmembrane helices by a knobs-into-holes packing characteristic of soluble coiled coils. Proteins Struct. Funct. Genet. 31:150-59

53. Leite J, Cascio M. 2001. Structure of ligand-gated ion channels: critical assessment of biochemical data supports novel topology. Mol. Cell. Neurosci. 17: 777-92

54. Lemmon MA, Engelman DM. 1994. Specificity and promiscuity in membrane helix interactions. Q. Rev. Biophys. 27:157-18

55. Levina N, Tötemeyer S, Stokes NR, Louis P, Jones MA, Booth IA. 1999. Protection of Escherichia coli cells against extreme turgor by activation of $\mathrm{MscS}$ and MscL mechanosensitive channels: identification of genes required for $\mathrm{MscS}$ activity. EMBO J. 18:1730-37

56. Levitt DG. 1978. Electrostatic calculations for an ion channel. I. Energy and potential profiles and interactions between ions. Biophys. J. 22:209-19

57. Li H, Cocco MJ, Steitz TA, Engelman DM. 2001. Conversion of phospholamban into a soluble pentameric helical bundle. Biochemistry 40:6636-45

58. Li M, Jan YN, Jan LY. 1992. Specification of subunit assembly by the hydrophilic amino-terminal domain of the Shaker potassium channel. Science 257: 1225-30

58a. Liu Y-S, Sompornpisut P, Perozo E. 2001. Structure of the KcsA channel intracellular gate in the open state. Nat. Struct. Biol. 8:883-87

59. Luecke H, Richter HT, Lanyi JK. 1998. Proton transfer pathways in bacteriorhodopsin at $2.3 \AA$ resolution. Science 280:1934-37 
60. MacKinnon R. 1991. Determination of the subunit stoichiometry of a voltageactivated potassium channel. Nature 350:232-35

61. Mattevi A, Rizzi M, Bolognesi M. 1996. New structures of allosteric proteins reveal remarkable conformational changes. Curr. Opin. Struct. Biol. 6:82429

62. Maurer JA, Elmore DE, Lester HA, Dougherty DA. 2000. Comparing and contrasting Escherichia coli and Mycobacterium tuberculosis mechanosensitive channels (MscL) - new gain of function mutations in the loop region. J. Biol. Chem. 275:22238-44

63. McDermott G, Prince SM, Freer AA, Hawthornthwaite-Lawless A, Papiz MZ, et al. 1995. Crystal structure of an integral membrane light-harvesting complex from photosynthetic bacteria. $\mathrm{Na}$ ture 374:517-21

64. Miyazawa A, Fujiyoshi Y, Stowell M, Unwin N. 1999. Nicotinic acetylcholine receptor at $4.6 \AA$ resolution: transverse tunnels in the channel wall. J. Mol. Biol. 288:765-86

65. Monod J, Wyman J, Changeux JP. 1965. On the nature of allosteric transitions: a plausible model. J. Mol. Biol. 6:306-29

65a. Morais-Cabral JH, Zhou Y, MacKinnon R. 2001. Energetic optimization of ion conduction rate by the $\mathrm{K}^{+}$selectivity filter. Nature 414:37-42

66. Murata K, Mitsuoka K, Hirai T, Walz T, Agre P, et al. 2000. Structural determinants of water permeation through aquaporin-1. Nature 407:599-605

67. Nagle JF, Tristram-Nagle S. 2000. Lipid bilayer structure. Curr. Opin. Struct. Biol. 10:474-80

67a. Nollert P, Harries WEC, Fu D, Miercke LJW, Stroud RM. 2001. Atomic structure of a glycerol channel and implications for substrate permeation in aqua(glycerol)porins. FEBS Lett. 504: 112-17

68. Ou XR, Blount P, Hoffman RJ, Kung C.
1998. One face of a transmembrane helix is crucial in mechanosensitive channel gating. Proc. Natl. Acad. Sci. USA 95:11471-75

69. Palczewski K, Kumasake T, Hori T, Behnke CA, Motoshima H, et al. 2000. Crystal structure of rhodopsin: a $\mathrm{G}$ protein-coupled receptor. Science 289: 739-45

70. Papazian DM, Shao XM, Seoh S-A, Mock AF, Huang Y, Wainstock DH. 1995. Electrostatic interactions of S4 voltage sensor in Shaker $\mathrm{K}^{+}$channel. Neuron 14:1293-301

71. Parsegian A. 1969. Energy of an ion crossing a low dielectric membrane: solutions to four relevant electrostatic problems. Nature 221:844-46

72. Partenskii MB, Jordan PC. 1992. Theoretical perspectives on ion-channel electrostatics-continuum and microscopic approaches. Q. Rev. Biophys. 25: 477-510

73. Pauling L, Corey RB. 1951. Configurations of polypeptide chains with favored orientations around single bonds: two new pleated sheets. Proc. Natl. Acad. Sci. USA 37:729-40

74. Pauling L, Corey RB, Branson HR. 1951. The structure of proteins: two hydrogenbonded helical configurations of the polypeptide chain. Proc. Natl. Acad. Sci. USA 37:205-11

75. Perozo E, Kloda A, Cortes DM, Martinac B. 2001. Site-directed spin-labeling analysis of reconstituted MscL in the closed state. J. Gen. Physiol. 118:193-206

76. Perutz M. 1990. Mechanisms of Cooperativity and Allosteric Regulation in Proteins. Cambridge: Cambridge Univ. Press

77. Popot J-L, Engelman DM. 2000. Helical membrane protein folding, stability, and evolution. Annu. Rev. Biochem. 69:881922

78. Rees DC, Chang G, Spencer RH. 2000. Crystallographic analyses of ion channels: lessons and challenges. J. Biol. Chem. 275:713-16 
79. Rees DC, DeAntonio L, Eisenberg D. 1989. Hydrophobic organization of membrane proteins. Science 245:510-13

80. Rees DC, Komiya H, Yeates TO, Allen JP, Feher G. 1989. The bacterial photosynthetic reaction center as a model for membrane proteins. Annu. Rev. Biochem. 58:510-13

80a. Riek RP, Rigoutsos I, Novotny J, Graham RM. 2001. Non- $\alpha$-helical elements modulate polytopic membrane protein architecture. J. Mol. Biol. 306:349-62

81. Roux B, Berneche S, Im W. 2000. Ion channels, permeation and electrostatics: insights into the function of KcsA. Biochemistry 39:13295-306

82. Russ WP, Engelman DM. 2000. The GxxxG motif: a framework for transmembrane helix-helix association. $J$. Mol. Biol. 296:911-19

83. Senes A, Gerstein M, Engelman DM. 2000. Statistical analysis of amino acid patterns in transmembrane helices: the GxxxG motif occurs frequently and in association with $\beta$-branched residues at neighboring positions. J. Mol. Biol. 296:921-36

84. Sewing S, Roeper J, Pongs O. 1996. $\mathrm{Kv}$ beta 1 subunit binding specific for Shaker-related potassium channel alpha subunits. Neuron 16:455-63

85. Shen NV, Pfaffinger PJ. 1995. Molecular recognition and assembly sequences involved in $\mathrm{K}^{+}$channel subunit proteins. Neuron 14:625-33

86. Sheng M, Laiao YJ, Jan YN, Jan LY. 1993. Presynaptic A-current based on heteromultimeric $\mathrm{K}^{+}$channels detected in vivo. Nature $365: 72-75$

87. Singer SJ. 1962. The properties of proteins in non-aqueous solvents. Adv. Protein Chem. 17:1-68

88. Sokolova O, Kolmakova-Partensky L, Grigorieff N. 2001. Three-dimensional structure of a voltage-gated potassim channel at $2.5 \mathrm{~nm}$ resolution. Structure 9:215-20

89. Spencer R, Chang G, Rees DC. 1999.
Feeling the pressure: structural insights into a gated mechanosensitive channel. Curr. Opin. Struct. Biol. 9:448-54

90. Stowell M, McPhillips TM, Rees DC, Soltis SM, Abresch E, Feher G. 1997. Light-induced structural changes in photosynthetic reaction center: implications for mechanism of electron-proton transfer. Science 276:812-16

90a. Sui H, Han B-G, Lee JK, Walian P, Jap BK. 2001. Structural basis of waterspecific transport through the AQP1 water channel. Nature 414:872-78

91. Sukharev S, Betanzos M, Chiang C-S, Guy HR. 2001. The gating mechanism of the large mechanosensitive channel MscL. Nature 409:720-24

92. Sukharev S, Durell SR, Guy HR. 2001. Structural models of the MscL gating mechanism. Biophys. J. 81:917-36

93. Sukharev SI, Blount P, Martinac B, Blattner FR, Kung C. 1994. A largeconductance mechanosensitive channel in $E$. coli encoded by $\mathrm{mscl}$ alone. Nature 368:265-68

94. Sukharev SI, Blount P, Martinac B, Kung C. 1997. Mechanosensitive channels of Escherichia coli: the MscL gene, protein, and activities. Annu. Rev. Physiol. 59:633-57

95. Sukharev SI, Schroeder MJ, McCaslin DR. 1999. Stoichiometry of the large conductance bacterial mechanosensitive channel of E. coli. A biochemical study. J. Membr. Biol. 171:183-93

96. Sukharev SI, Sigurdson WJ, Kung C, Sachs F. 1999. Energetic and spatial parameters for gating of the bacterial large conductance mechanosensitive channel, MscL. J. Gen. Physiol. 113:52539

97. Sussman J, Lin D, Jiang J, Manning N, Prilusky J, et al. 1998. Protein Data Bank (PDB): database of three-dimensional structural information of biological macromolecules. Acta Crystallogr. D 54: 1078-84

98. Tiwari-Woodruff SK, Schulteis CT, 
Mock AF, Papazian DM. 1997. Electrostatic interactions between transmembrane segments mediate folding of Shaker $\mathrm{K}^{+}$channel subunits. Biophys. J. 72:1489-500

99. Toyoshima C, Nakasako M, Nomura H, Ogawa H. 2000. Crystal structure of the calcium pump of sarcoplasmic reticulum at $2.6 \AA$ resolution. Nature 405:647-55

100. Toyoshima C, Unwin N. 1988. Ion channel of acetylcholine receptor reconstructed from images of postsynaptic membranes. Nature 336:247-50

101. Tsukihara T, Aoyama H, Yamashita E, Tomizaki T, Yamaguchi H, et al. 1996. The whole structure of the 13-subunit oxidized cytochrome $c$ oxidase at $2.8 \AA$. Science 272:1136-44

102. Ubarretxena-Belandia I, Engelman DM. 2001. Helical membrane proteins: diversity of functions in the context of simple architecture. Curr. Opin. Struct. Biol. 11:370-76

103. Unwin N. 1995. Acetylcholine receptor channel imaged in the open state. Nature 373:37-43

104. Wallin E, Tsukihara T, Yoshikawa S, von Heijne G, Elofsson A. 1997. Architecture of helix bundle membrane proteins: an analysis of cytochrome $c$ oxidase from bovine mitochondria. Protein Sci. 6:808-15

105. White SH, Wimley WC. 1999. Membrane protein folding and stability: physical principles. Annu. Rev. Biophys. Biomol. Struct. 28:319-65

106. Yi BA, Jan LY. 2000. Taking apart the gating of voltage-gated $\mathrm{K}^{+}$channels. Neuron 27:423-25

107. Yoshimura K, Batiza A, Schroeder M, Blount P, Kung C. 1999. Hydrophilicity of a single residue within MscL correlates with increased channel mechanosensitivity. Biophys. J. 77:1960-72

108. Zhou M, Morais-Cabral JH, Mann S, MacKinnon R. 2001. Potassium channel receptor site for the inactivation gate and quaternary amine inhibitors. Nature 411:657-61

108a. Zhou Y, Morais-Cabral JH, Kaufman A, MacKinnon R. 2001. Chemistry of ion coordination and hydration revealed by a $\mathrm{K}^{+}$channel-Fab complex at $2.0 \AA$ resolution. Nature 414:43-48 


\section{CONTENTS}

Frontispiece-George Feher

xviii

MY ROAD TO BIOPHYSICS: PICKING FLOWERS ON THE WAY TO PHOTOSYNTHESIS, George Feher

The Natural History OF Protein Domains, Chris P. Ponting and Robert R. Russell

MaGnetic Resonance STUdies OF THE BACTERIORHODOPSIN PUMP CYCLE, Judith Herzfeld and Jonathan C. Lansing

FLOW CYTOMETRIC ANALYSIS OF LIGAND-RECEPTOR INTERACTIONS AND MOLECUlAR ASSEMBLIES, Larry A. Sklar, Bruce S. Edwards, Steven W. Graves, John P. Nolan, and Eric R. Prossnitz

STRUCTURAL AND THERMODYNAMIC CORRELATES OF T CELL SignAling, Markus G. Rudolph, John G. Luz, and Ian A. Wilson

PIP $_{2}$ AND PROTEINS: INTERACTIONS, ORGANIZATION, AND INFORMATION FLOW, Stuart McLaughlin, Jiyao Wang, Alok Gambhir, and Diana Murray

NMR STUDIES OF LIPOPROTEIN STRUCTURE, Robert J. Cushley and Mark Okon

THE $\alpha$-HeliX AND THE ORGANIZATION AND GATING OF CHANNELS, Robert H. Spencer and Douglas C. Rees

The LinKage BetweEn PROTEIN Folding AND FunCTIONAL COOPERATIVITY: Two Sides OF THE SAME CoIn?, Irene Luque, Stephanie A. Leavitt, and Ernesto Freire

The SeARCH AND Its OUTCOME: High-Resolution STRUCTURES OF RIBOSOMAL PARTICLES FROM MESOPHILIC, THERMOPHILIC, AND HALOPHILIC BACTERIA AT VARIOUS FUNCTIONAL STATES, Ada Yonath

PRINCIPLES AND BIOPHYSICAL APPLICATIONS OF LANTHANide-BASED PROBES, Paul R. Selvin

Single-PARTICLE IMAGING OF MACROMOLECULES BY CRYO-ELECTRON MiCROSCOPY, Joachim Frank

FORCE EXERTION IN FUNGAL INFECTION, Martin Bastmeyer, Holger B. Deising, and Clemens Bechinger 
The PAPILlomavirus E2 Proteins: StRUCTURE, FunCtion, AND BIOLOGY, Rashmi S. Hegde

CONFORMATIONAL DYNAMICS OF THE CHROMATIN FIBER IN SOLUTION: DETERMINANTS, MECHANISMS, AND FUNCTIONS, Jeffrey C. Hansen

Paramagnetic Resonance of Biological Metal Centers, M. Ubbink, J. A. R. Worrall, G. W. Canters, E. J. J. Groenen, and M. Huber

Computational Cell Biology: SPatiotemporal Simulation of Cellular Events, Boris M. Slepchenko, James C. Schaff, John H. Carson, and Leslie M. Loew

RHODOPSIN: INSIGHTS FROM RECENT STRUCTURAL STUDIES, Thomas P. Sakmar, Santosh T. Menon, Ethan P. Marin, and Elias S. Awad

CONFORMATIONAL REGULATION OF INTEGRIN STRUCTURE AND FunCTION, Motomu Shimaoka, Junichi Takagi, and Timothy A. Springer

INDEXES

Subject Index

Cumulative Index of Contributing Authors, Volumes 27-31 541

Cumulative Index of Chapter Titles, Volumes 27-31

\section{ERRATA}

An online log of corrections to Annual Review of Biophysics and Biomolecular Structure chapters may be found at http://biophys.annualreviews.org/errata.shtml 